
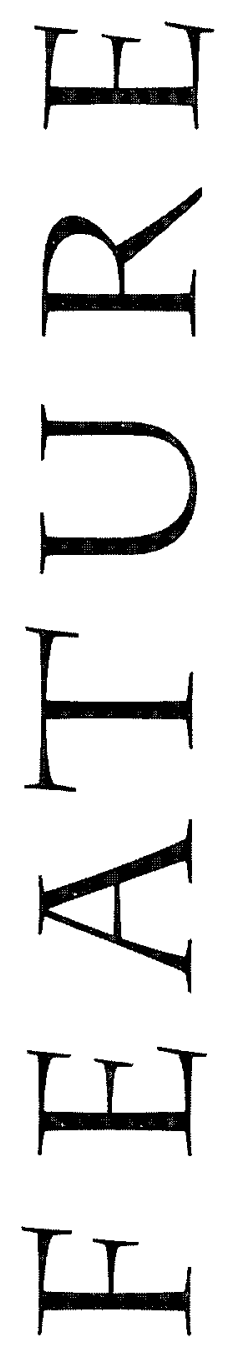

\title{
Gaining Links: Health Information Networks Arise with Integration Challenges
}

As automation moves healthcare toward the twenty-first century, health information networks are creating relationships among many diverse types of practitioners.

\section{MARY JANE GORE}

A variety of technological advances has yielded new, exquisitely engineered Community Health Information Networks (CHINs). Through CHINs, pharmacists and other healthcare professionals can share clinical data, as well as financial, inventory, and other nonclinical data. This article will examine some of the trends in information exchange across enterprises and communities and discuss automation of pharmacy services.

\section{COMMUNITY HEALTH} INFORMATION NETWORKS

CHINs comprise engineered electronic infrastructures that allow information exchange across complicated organizations, communities, or regions. According to Peter Keen, author of Competing for Time, a health information network is a multiservice system onto which a growing variety of services can be added at a reasonable, incremental cost.

In March 1993, there were more 
Figure 1. WHIN functionalitya

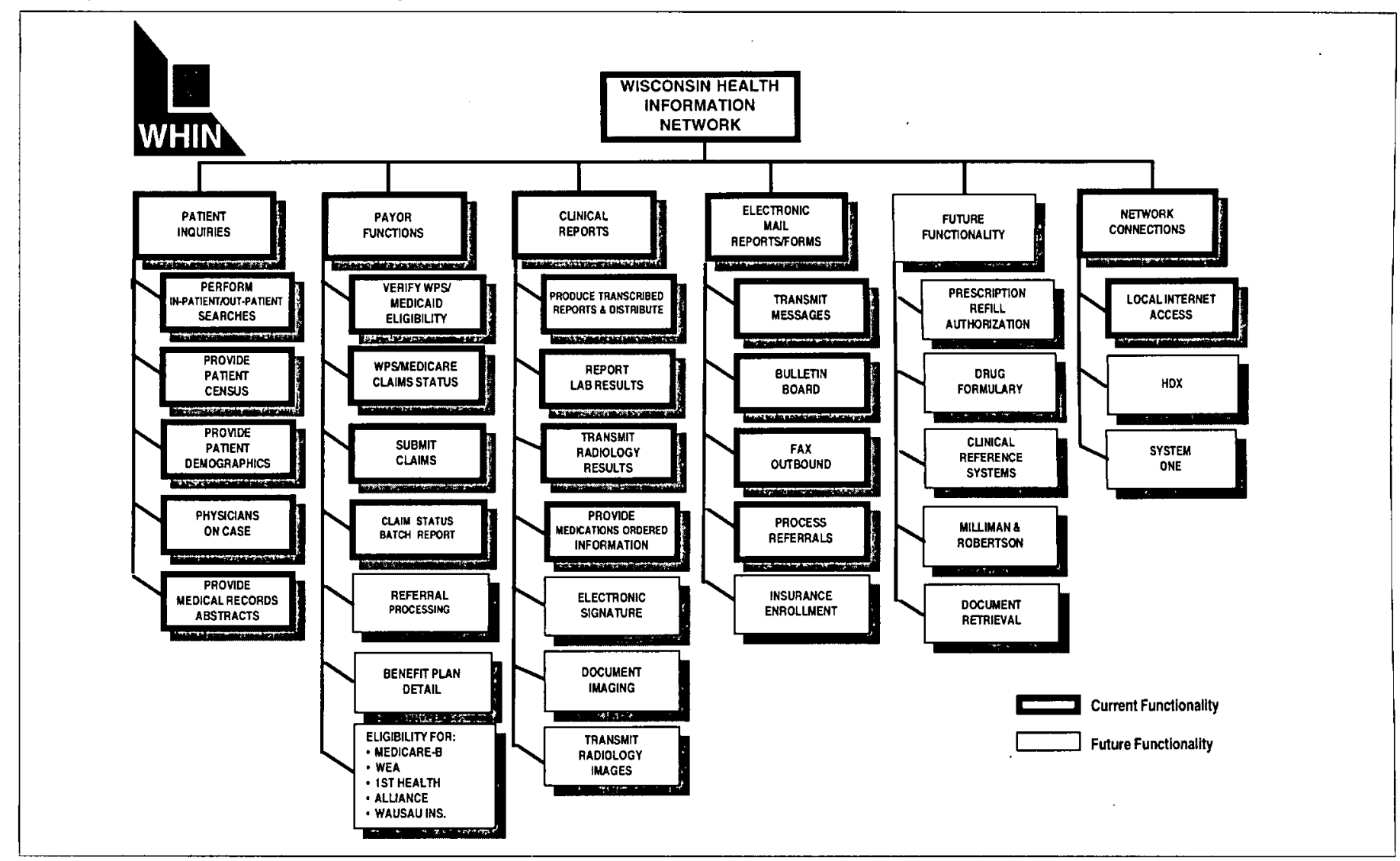

a Courtesy of Wisconsin Health Information Network

than 30 of these information networks around the country, according to Carolyn Dunbar, editor of Computers in Healthcare (now called Health Systems Technology). Now that number is closer to 70 , says a health information technology expert who recently attended a seminar on CHINs. This number, however, also includes concepts for CHINs that are not yet implemented.

One of the best known examples is the Wisconsin Health Information Network (WHIN), a statewide network located in Brookfield with the majority of users in southeast Wisconsin. WHIN is "a good 1.8 months ahead" of any other regional health information network in terms of the degree of implementation of services it offers to users, says Rhondä Nelson, WHIN's vice president of sales.

Through the WHIN, medical professionals are able to (Figure 1):

$\nabla$ Exchange clinical data, including information in the form of medica- tions ordered, laboratory results, radiology results, and medical reports and transcriptions;

$\checkmark$ Exchange financial data (e.g., submitting claims and transmitting claim status batch reports or verifying Medicaid eligibility);

$\checkmark$ Use services that are now coming online, such as a prescription refill authorization service with independent pharmacists; and

$\nabla$ Access services from remote locations-for example, a physician checking pharmacologic records or laboratory results from home

The evolution of WHIN typifies the growth palterns of a CHIN. WHIN began with a "proprietary physician-hospital link...then expanded to include access to data at other, competing hospitals....I soon broadened its sponsorship to include other stakeholders in the healthcare delivery system, including employers, payers, and government agencies. WHIN quickly adopted a phi- losophy of steadily adding incremental value, and is now in the process of linking with larger national and international networks, including the Internet." 1

Costs for the service are between $\$ 60,000$ and $\$ 120,000$ annually for a hospital with $1.50+$ beds, according to Frank Hoban, general manager of the WHIN 2

\section{PHARMACY: A CORE SERVICE IN CHINS}

Pharmacies are one of the eight major sites served through the WHIN network; others are payers, banks, medical information services, employers, testing organizations (laboratory and imaging organizations), hospitals, and physicians.

WHIN is just now establishing pilot sites to test the prescription refill authorization capabilities of the network. "We're really at the front end of bringing anybody live with this capability," Nelson says. WHIN is using AIM-RITE 
pharmacy practice management software by F. Dohmen Co., a wholesale drug company with a software development division.

The new system will allow pharmacists and physicians to spend less time communicating by telephone and more time interacting with patients, according to Bob Wendland, president and CEO of Dohmen, which is headquartered in Germantown, Wis. Physicians will be able to order prescription refills by tapping a computer keyboard. Future applications for pharmacy may include prescription orders, obtaining patients' pharmacy records, drug information updates, access to pharmacy claim records, and access to pharmacy health benefit plan formulas, according to WHIN literature.

Restat, a Dohmen company division based in West Bend, Wis., provides data and prescription drug management services across the country to self-insured employers, insurers, and healthcare purchasing cooperatives. ${ }^{3}$ Dohmen executives hope that the WHIN service will complement the services of its Restat division. ${ }^{3}$

"The logical pharmacies to come on first will be those pharmacies that have the F. Dohmen software installed," Nelson says. She says that WHIN was also in contract negotiation with Walgreen's pharmacy network, to connect that network to WHIN, but apparently Walgreen's is selling its system and IBM was to be the buyer, so negotiations with WHIN have halted.

"In time, the goal is to create a common user interface that would connect all pharmacies for refill authorization," Nelson says. WHIN has developed some templates that work as the electronic forms to send from physician to pharmacy, in the cycle of refill authorization. These forms can be communicated from any WHIN user to any pharmacy user tied to WHIN, Nelson says.

\section{HEALTHY SKEPTICISM IN ORDER}

David E. Garets, chief information officer of the 165-bed Magic Valley Regional Medical Center in Twin Falls,
Idaho, is leading an effort to assemble a $\mathrm{CHIN}$ for the 134,000-square-mile, 182,000-person region that his hospital dominates. Of the other five hospitals in the consortium, one has a daily census of two beds.

The effort that began with the MVRMC will soon encompass five other hospitals, pharmacies, physician offices, dental offices, nursing homes, home health agencies and other caregiving agencies, private insurers, $\mathrm{HMOs}$, and governmental reporting agencies. ${ }^{4}$ To meet its goal of communitywide health (known as Healthy Magic Valley 2012), the creators hope to use the CHIN to track the success of community health strategies. Right now, the coalition of hospitals is surveying and ranking health programs and assembling information on leading causes of hospital admissions-heart disease, cancer, labor and delivery, diabetes, and preventable injuries-to track four to five major health problems.

Garets has been observing the progress of many CHINs across the country. "The CHIN in Wisconsin is limited in its scope, as is the CHIN in the Puget Sound area," says Garets, who has had conversations with leaders of the Puget Sound-area CHIN. "They are just getting into clinical systems now, and it is complicated."

Chicago's CHIN, which comprises about 75 hospitals, each with its own hospital information system, is trying to determine how to share clinical information. "Most people are just passing eligibility information and billing information," he says. "The data have been financial rather than clinical. It is an overwhelming task to integrate the disparate, proprietary information systems that hospitals have."

The Magic Valley project in Idaho has an advantage in that none of the hospitals in question yet has a sophisticated clinical information system that would create a virtual patient repository of data from all points of care. For some of the hospitals, the CHIN computer system is the first clinical system the hospital would have.

Garets explains other ways in which the Idaho project is different from others. Idaho effectively has no managed care yet, because the state is so sparsely populated; discounted feefor-service is becoming common. The Idahoan hospitals and healthcare providers, including pharmacies, are individual institutions, with no ties whatsoever, as opposed to Sisters of Providence in Portland, Oregon, and many other systems nationwide that tie together physicians whose practices are owned by the multihospital system. A third difference is that the Magic Valley collaborative group is tying public health to its CHIN.

"No one else in the country is doing that, which I think is an oversight," Garets says. "The only reason we thought of it is that the head of our state public health department is on the board of directors of this hospital. She'll say, 'How do you know who's doing immunizations for kids? Pediatricians don't know what public health has done and vice versa.' And there are many more good examples of why we need to include public health."

Garets has had contact from people in many other rural areas who are looking to Idaho as a role model of information management. Money is the obstacle. "But even the way we've worked it out, a 30-bed hospital will end up spending $\$ 2,000$ and getting a clinical information system out of it," Garets says. "That is cheap."

Because healthcare providers everywhere must prove patient outcomes relative to costs, no one can afford to be without a good clinical information system. Even though ldaho is largely new to managed care, discounted fee-for-service has arrived, (e.g., a hospital gets a group of employees in exchange for a reduced rate). Without the proper data, "how do you know if you are making money at $25 \%$ of usual fees? How can you reduce costs, how do you re-engineer your processes to get waste out of it?" Garets asks rhetorically.

Well-organized, accessible information is a major antidote, along with controls on physician activities. "In a hospital, the physician controls $85 \%$ of ex- 
penses with their pens," Garets says. He cites the example of Intermountain Healthcare in Salt Lake City, Utah, where physicians can tap into a database that will give them immediate cost-effectiveness information and choices of drugs to prescribe.

Nationwide, most of the CHINs are being funded by insurance money, sometimes through established foundations, in addition to the member fees, Garets says. To date, the Idaho project has to rely on the kindness of others in the form of grant money-a total of $\$ 9$ million is needed for two years of the project. Two HIS companies are finalists for installing the actual clinical information system, and they may be able to apply their work in other rural settings, Garets predicts.

\section{SECURITY AND \\ CONFIDENTIALITY CONCERNS}

Ironically, the drawback to CHINs is also its strength-access to data. The biggest asset that insurance companies own is their data on individual patients and on patient populations, and they don't want their data to fall into the hands of competitors. Security is a problem with regard to both patient and plan information, says Terri Bernacchi, manager of pharmacy services for CompCare, an HMO in Milwaukee. CompCare has not yet joined WHIN, although management is considering joining up with the network, Bernacchi says.

Raymond D. Aller, M.D., a professor of pathology and of medical informatics at the University of Utah School of Medicine, notes that security is one of the major themes of medical informatics. "Confidentiality and security need to be part of your way of life, it is not a module that solves your problems. It needs to be part of the medical culture and a part of this electronic culture as well," he says. Inherently, electronic records can be made more secure than paper records, with passwords and proper protections, but this is not automatic, Aller cautions.

The various WHIN systems are structured so that personnel with pass- words can obtain only certain information. For example, an insurance claims processor might only have a password that allows him to look at insurance data, but not medical data. Likewise, staff in a physician's office may be able to search laboratory data by patient name, rather than have access to the universe of laboratory data.

Garets says that there is a reluctance on the part of physicians and others to share some clinical informationsome of it will not make its way onto the CHIN he is working to build, he says. History of alcoholism or mental status may be available to the primary care physician, for example, but the information may stop there, in some cases. Garets agrees that confidentiality is a major obstacle to implementation.

\section{WHO BENEFITS FROM CHINS?-}

With some concerns about security and confidentiality, most sources interviewed agreed that $\mathrm{CHINs}$ will reduce duplicative services and expand the medical knowledge base in beneficial ways.

Garets notes that he was "appalled" by the lack of automation in the healthcare industry, after he left his information management job at AT\&T after 13 years. "[Healthcare providers] fly by the seat of their pants, and physicians make educated guesses," he told JMCP. "I have been trying to apply the same kinds of information management to this hospital as has been applied to other industries for years, like banks or manufacturing companies. You can go to any ATM in the country and get money, but if you show up in an ER that's not in your hometown, they don't have the faintest idea about the information that might help save your life." Patient records, the "database" of hospitals, are merely "fat file folders tucked into a file drawer" in the medical records divisions of many institutions, he complains.

Terri Bernacchi says that CHINs could make much more information available to providers. "A physician writing a prescription can have access to formulary and drug benefit informa- tion, the pharmacist has access to that information, and the payer has all of the checks and screens to prevent off-benefit use before it happens," Bernacchi told JMCP.

From the payer perspective, a CHIN helps to clarify medical information that is difficult, if not impossible, to obtain. For example, payers often do not know whether Prozac is being used to treat depression or mood disorders. By linking the patient's medical history and record to the CHIN, that information could become available.

"As a pharmacist who has worked in an inpatient world and an outpatient world without all of that information [on a CHIN], sometimes you can't make good interventions because you don't know enough," Bernacchi says. The laboratory test information that may show drug toxicity also would be available to the pharmacist who is operating in the dark on the medical side, she adds.

CHINs would also help to reduce the amount of duplicate utilization, Bernacchi says. She gives the example of a physician who orders a urinalysis for a patient. The patient is referred to a urologist. If the urologist had not received the records by the time he or she sees the patient, another urinalysis might be ordered. Through a health information network, however, the urologist could tap into the system and obtain the results from the other physician's office. An entire CHIN multiplies the efficiencies of automation, Bernacchi says. "The CHIN could reduce unnecessary testing, unnecessary drugsit has applications in every specialty."

$\square \quad$ References

1. Skjei E. Healthcare takes on the CHIN. CAP Today 9(6):1995:1,32-35

2. Jensen D. Network puts providers in data fast lane; some roadblocks loom. Milwaukee Sentinel, Focus section: 1993(Dec. 11).

3. Daykin T. State network to link pharmacies, doctors. Milwaukee Sentinel: 1995(Jan. 28).

4. Kennedy M. Planning a Community Health Network: an interview with David E. Garets and Cheryl Juntunen. J Qual Improve 1994;

20(11):651-6. 